\title{
The Design of Flower Ecological Environment Monitoring System Based on ZigBee Technology
}

\author{
Xiaoqing Guo ${ }^{1, *}$ and Xinjian Xiang ${ }^{2}$ \\ ${ }^{1}$ School of Biological and Chemical Engineering, Zhejiang University of Science and \\ Technology, Hangzhou, Zhejiang Province, P.R. China 310023 \\ Tel.: 0571-85070376 \\ hzguoxiaoging@163.com \\ ${ }^{2}$ School of Automation and Electrical Engineering, Zhejiang University of Science and \\ Technology, Hangzhou, Zhejiang Province, P.R. China 310023
}

\begin{abstract}
Ecological environment is the key point of improving the flower's quality and quantity. Due to China's flower production management at a lower level, there is no scientific method in real-time monitoring of the flower's ecological environment. In order to solve the problem such as high costs; poor monitoring point scalability, poor mobility and other issues in traditional flower basement's data acquisition system, this paper devises a wireless real-time system based on ZigBee technology for the monitoring of flower's ecological environment. By the analysis of ZigBee technology's characteristics, it focuses on the design of wireless gateway with $\mathrm{S} 3 \mathrm{C} 4510 \mathrm{~B}$; wireless sensor node control module AT89S51 and the communication module CC2430; analyses the Zigbee protocol stack network's formation and designs data acquisition and communication procedures. By monitoring every flower's ecological environment indicators in practice, this system can meet the needs of the real-time monitoring for flower's ecological environment.
\end{abstract}

Keywords: Flower basement; eco-environmental monitoring; Zigbee; wireless sensor network; data acquisition.

\section{Introduction}

China is the world's largest producer of flowers, flower production occupies an important share of the national economy, but in the current production management, there is no scientific method in real-time monitoring of the flower's ecological environment such as growth conditions, cultivation and prevention of pests and diseases. (Ji Qing et al., 2007) Wireless sensor network (WSN) is consist of a large number of low-cost micro-sensor node with communication, sensing and computing deployed in the monitoring region, through self-organization constitutes a "smart" monitoring and control network (Jiang Ting et al., 2006). University of California, Berkeley used Micromote nodes to deployed wireless sensor network in a $70 \mathrm{M}$ red cedar for monitoring

\footnotetext{
* Corresponding author.
} 
its environment change. Intel used Crossbow's Mote nodes in the deployment of WSN in a vineyard to monitor small changes in environment (Li Xiaomin et al., 2007). These studies provide an effective reference to the real-time monitoring for flower's ecological environment.

Zigbee with star, mesh, tree-like network topology, can be used in the WSN network and other wireless applications. ZigBee works in the $2.4 \mathrm{GHz}$ license-free frequency band can accommodate up to 65,000 nodes. Those nodes with low-power consumption can work 6 to 24 months by two AA batteries (Liang Yufen et al., 2007). Besides, it has a higher degree of reliability and security. These advantages of ZigBeebased wireless sensor networks are widely used in industrial control, consumer electronics devices, automotive electronics, home and building automation, medical equipment control(Liu Yongqiang et al., 2007).

For the effective monitoring flower ecological environment, improve the production and management process, and promote high-yielding flowers and eugenics, this paper devises a wireless real-time system based on ZigBee technology for the monitoring of flower's ecological environment, realized the real-time monitoring of flower ecological factors such as temperature, humidity, light, as well as nutritional status, provided technical support for the best fostering strategy and scientific management.

\section{System Design}

At present, many companies have introduced their Zigbee wireless development system based on CC2430. Because of its low-cost, this paper choose protocol stack source code and development kit support Zigbee protocol standards provided by TI company. Flower ecological environment with complex environment variable need the network routing and data fusion capabilities, and any network structure are based on a simple star structure. This star structure is also studied in this paper. A number of Zigbee terminal nodes (RFD) and a Zigbee Coordinator (FFD) compose a star-WSN, RFD sensors collect environmental parameters (temperature, humidity and light, etc.), and send to the FFD through the network, finally feedback PC by the serial interface. WSN nodes including RFD and FFD, with MCU and RF chip mode to meet the complex monitoring stability and applicability of the ecological environment. Each node is consisting of data acquisition sensors, processing module, wireless communication module and power module.

\subsection{Data Acquisition Module}

Temperature, moisture and light are important factor in the monitoring region. This paper take temperature for example, introduced the WSN data acquisition module. DS18B20 digital temperature sensor with 12-bit Celsius temperature measurements, it communicates over a 1 -Wire bus and has an operating temperature range of $-55^{\circ} \mathrm{C}$ to $+125^{\circ} \mathrm{C}$. DS18B20 includes 3-pin grounded (GND), data input / output pins (DQ) and power line (VDD), as well as two power supply mode parasitic capacitance and external $+5 \mathrm{~V}$ power. In the paper, access from the VDD power supply pin make the DQ does not need to strengthen the pull, so the bus controller does not keep high in the temperature conversion and allow the data exchanges, GND pin can not be left vacant. 


\subsection{Microprocessor and RF Module}

Microprocessor as the key unit finishes data collection, processing and delivery with other units. Zigbee protocol stack on the system require 8-bit microprocessor; fullfunction device (FFD) node of the protocol stack, ROM $<32 \mathrm{k}$; simple function device (RFD) node protocol stack, ROM about 6k; RFD also need to have sufficient RAM to save node binding table, found table and routing table. AT89S51 as microprocessor and CC2430 as RF chip had been selected.

\section{Software of Wireless Data Acquisition Node}

\subsection{Data Transmission Networks}

FFD and RFD initialization procedure both include hardware initialization, Zigbee network initialization and serial communications initialization, call the stack function ZigBee_Init () and Console_Init () to complete Zigbee agreement and serial communications initialization, at the same time call application-layer function Hardware_Init () to complete hardware initialization. Define the current Primitive to track network communication primitives, at the beginning of program running, current Primitive mean NO-PRIMITIVE, when it finish other primitives, it mean NO-PRIMITIVE again.

\subsubsection{RFD Nodes Software Flowchart}

Top layer send NLME-NETWORK-FORMATION request to network protocol layer for establish a new network. After the establishment of network, network layer will ask MAC layer for the energy detection scan to the channel that protocol or physical layer provides. Successfully received the results of energy detection scans, it will sort the channel with increased energy and discard those channel beyond their energy range, finally choose the allowed channel. If the network establish success, report to the Top layer through NLME-NETWORK-FORMATION confirm, else RFD will connect MAC layer device's network. MAC layer using the primitive MLME ASSOCIATE indication initialization, if the network allows devices to join, MLME - ASSOCIATE response its primitive state. At last, MAC layer send a successful response to network layer, and network layer report to RFD. RFD sends inquiries key information to determine whether there is any terminal node to send information.

\subsubsection{FFD Nodes Software Flowchart}

After initialization, first of all to determine whether the equipment has joined the network. If FFD already joined the network, send a message directly; If have not yet joined the network, it will be treated as a solitary point to join the original network, or the network layer rescan the new network; if joined a new network, the application layer send NLME-NETWORK-DISCOVERY request primitive to bottom layer. The primitive includes scan channel parameter and time parameter, network layer receive the primitive and ask MAC layer to run scan program, feedback the scan result to application layer, then application layer will connect one of those networks and send NLME-JOIN request to the network layer. Network layer find o a suitable father device from its neighbor table, and send MLME-ASSOCITION request to MAC layer 
for a connection. The result will back through MLME-ASSOCITION confirm primitive. If FFD join network as a solitary point, send NLME-JOIN request to network layer for a connection. If already joined network, it would start the environment parameter conversion and send information such as temperature and humidity to RFD which ask for data query.

\subsection{Temperature Acquisition Software Flowchart}

DS18B20 has a 1-Wire bus architecture, temperature conversion and read/write need follow basic rules by writing command in ROM (write $44 \mathrm{H}$ mean start the temperature conversion once; write BEh mean read the register). The data acquisition process is function row_reset () as sensor reset and initialization; function write_byte () write command to the ROM for next temperature conversion or read/write; function read_byte () read the register which save the temperature value and send the value to wireless protocol stack.

\section{System Testing}

After finish the program write to the RFD and FFD, connect to computer by RS-232, using serial debugger software as screen output on computer. The software baud set at 19200 bps with 8-bit data bit, 1 stop bit, no parity-tested and data flow control bit. Then power the RFD devices, they will search the channel to establish network, if done, display 4 bit data with 16-band, which means the new network number. Now RFD allows FFD join the new network. Power the FFD devices and wait until the network address display on the screen, which means the whole network establish successfully.

Once the network established, push button at the RFD device to send the inquire command, when the FFD device receive this command, it start the temperature conversion and send the temperature value back to RFD device, which output: "Received 25.0" on the computer by RS-232. "25.0" is the region temperature where set those RFD and FFD devices, the wireless temperature acquisition finished. Other devices in the star network work as the same way finally send their region's environment parameters and complete the multi-point real-time acquisition.

\section{Conclusion}

Modern agricultural production needs the support from science and technology. In this paper, AT89s51 MCU and CC2430 RF chip complete the wireless sensor networks with star topology. Zigbee protocol stack complete the data exchange and network management by the primitive used in each layers. RFD sensors collect flower basement's environment parameters and send from application layer to physical layer by primitives, through wireless physical channel to the FFD device, completed the wireless real-time acquisition. The design provides a simple low-cost solution for wireless data acquisition, an effective method for monitoring flower environment parameters and flower growth, a great support for flower cultivation in decision-making 
and production management. It's an agricultural automation system example, with the agricultural scale development, this agricultural automation system based on wireless network technology has a very broad application prospects.

\section{Acknowledgements}

This material is based upon work funded by Zhejiang Provincial Natural Science Foundation of China under Grant No. Y108268.

\section{References}

Qing, J., Peiyong, D.: Study and implementation of a wireless sensor network based on zigbee technology. Sensor World (10), 30-35 (2007)

Ting, J., Chenglin, Z.: Zigbee technology and application, pp. 167-237. Press of Beijing University of Posts and Telecommunications (2006)

Xiaomin, L., Zhihong, Z., Zhi, G., et al.: Study and experiment on zigbee wireless sensor network. Electronic measurement technology 30(6), 133-137 (2007)

Yufen, L., Deyun, G., Yanchao, N., et al.: Review of the application system of WSN. Application of electronic technology (9), 3-9 (2007)

Yongqiang, L., Bing, Z.: Environmental monitoring for the design of wireless sensor network node. Popular Science \& Technology 99(5), 46-47 (2007) 